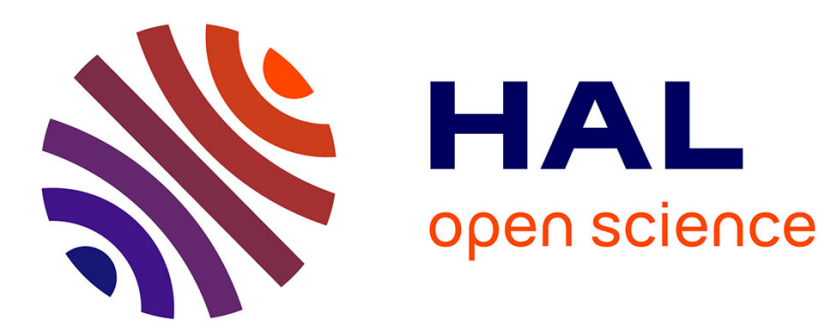

\title{
On a Markov chain model for population growth subject to rare catastrophic events
}

\author{
Thierry Huillet
}

\section{To cite this version:}

Thierry Huillet. On a Markov chain model for population growth subject to rare catastrophic events. Physica A: Statistical and Theoretical Physics, 2011, 390 (Issue 23-24), pp 4073-4086. hal-00602762

\section{HAL Id: hal-00602762 \\ https://hal.science/hal-00602762}

Submitted on 23 Jun 2011

HAL is a multi-disciplinary open access archive for the deposit and dissemination of scientific research documents, whether they are published or not. The documents may come from teaching and research institutions in France or abroad, or from public or private research centers.
L'archive ouverte pluridisciplinaire HAL, est destinée au dépôt et à la diffusion de documents scientifiques de niveau recherche, publiés ou non, émanant des établissements d'enseignement et de recherche français ou étrangers, des laboratoires publics ou privés. 


\title{
ON A MARKOV CHAIN MODEL FOR POPULATION GROWTH SUBJECT TO RARE CATASTROPHIC EVENTS
}

\author{
THIERRY E. HUILLET
}

\begin{abstract}
We consider a Markov chain model for population growth subject to rare catastrophic events. In this model, the moves of the process are getting algebraically rare (as from $x^{-\lambda}$ ) when the process visits large heights $x$, and given a move occurs and the height is large, the chain grows by one unit with large probability or undergoes a rare catastrophic event with small complementary probability $\sim \gamma / x$. We assume pure reflection at the origin. This chain is irreducible and aperiodic; it is always recurrent, either positive or null recurrent.

Estimates are obtained for first-return time probabilities to the origin (excursion length), eventual return (contact) probability and excursion height. All exhibit power-law decay in some range of the parameters $(\gamma, \lambda)$. We show a scaling relationship between heights and lengths of the excursions. From this, the mean and median of both the empirical average and sample maximum are shown to grow algebraically with exponents being identified in terms of $(\gamma, \lambda)$.
\end{abstract}

Keywords: Population growth, Markov chain, catastrophic events, height and length of excursions, scaling.

PACS 87.23.Cc, 02.50.Ey

MSC primary $60 \mathrm{~J} 10$, secondary $42 \mathrm{C} 05$

\section{INTRODUCTION}

We study a particular instance of a discrete-time Markov chain subject to rare catastrophic events or disasters. This model is in the class of the "house-of-cards" processes as the authors of ([11], p. 47) and ([12], p. 9) suggestively call them. In contrast to most studies on similar subjects in the literature (also often dealing with continuous times), we limit ourselves to the case where only birth or holding events can occur at times when disasters are ruled out. See [3], [5], [13] and [21].

Our model can precisely be described as follows.

Let $\lambda \geq 0, \gamma>0$. Consider the following Markov chain $\widetilde{X}$ on $\mathbb{Z}_{+}=\{0,1, \ldots\}$ : Given $\widetilde{X}_{n}=x \in\{1,2, \ldots\}$, the increment of $\widetilde{X}_{n}$ is

$$
\begin{aligned}
&+1 \text { with probability : } \widetilde{p}_{x}=x^{-\lambda} e^{-\gamma / x} \\
& 0 \text { with probability : } \widetilde{r}_{x}=1-x^{-\lambda} \\
&-x \text { with probability : } \widetilde{q}_{x}=x^{-\lambda}\left(1-e^{-\gamma / x}\right) \sim \gamma / x^{\lambda+1} .
\end{aligned}
$$

$\lambda>0$ accounts for the fact that moves of $\widetilde{X}$ get rare when $x$ gets large, and given a move occurs and $x$ is large, $\widetilde{X}$ grows by one unit with large probability $e^{-\gamma / x}$ or 
undergoes a rare catastrophic event with small complementary probability $\sim \gamma / x$. We assume pure reflection at the origin. This chain is irreducible and aperiodic; it is always recurrent, either positive or null recurrent.

The birth and holding probabilities depend on the current state of the population in the specific way just described. A model with a similar flavor can be found in [17] although the Authors deal here with continuous-time birth and death processes, which is of course not our case. The study of stochastic stability of the model with long-range-dependence and power-law crash sizes developed in [19] seems to be the closest to our own purposes.

Our study includes first-return time probabilities to the origin (excursion length), eventual return (contact) probability to the origin, excursion height, time to failure and estimates of the growth of the mean and median of both the empirical average and sample maximum. It is based on a scaling relationship between excursions heights and lengths. Because catastrophic events are assumed to be rare, the population growth is shown to be slow and in fact algebraic in the number of steps. We identify the algebraic exponents of growth in terms of $(\gamma, \lambda)$.

\section{Motivations AND RELATION TO SIMILAR WORKS IN THE LitERATURE}

Before we proceed with the mathematical study of our specific model, let us briefly give some motivations together with the mentioning of some relations to the vast existing literature on growth-collapse models. Growth-collapse processes where long periods of growth are interrupted by rare crash events occur in a large variety of systems. For instance in applications related to Transmission Control Protocol (see [19] for a precise description) or, as we will explain it in more details in the next Section, in the context of machine replacement (see [18]) in Queuing Systems. But also in the Physics of Self-Organized-Criticality such as sandpile growth processes where a long accumulation of grains is brutally interrupted by the occurrence of a critical avalanche (see [1]). Self-Organized-Criticality where an energy accumulation occurs preceding a rapid energy redistribution (or stress-release) is also suspected to explain power-law distributions of earthquakes sizes (see [2], [6] and [7]). And also, as we do here typically, growth-collapse models occur as models for population growth subject to rare catastrophic extinction events. Not to briefly mention crashes in financial mathematics.

As we mentioned in the Introduction, most works deal with continuous times, see for instance [4]. In several studies, the growing part of the process is more complicate than ours; for instance in [8] and [16], the Authors consider it as a one-sided Lévy subordinator with times and sizes of the crashes depending on the system's state. In [9], similar ideas are applied to model the charge and discharge of a capacitor. In [10], the inflow process undergoes a smooth deterministic growth/decay evolution with perturbations designed in such a way that the overall process obeys a geometric Langevin equation driven by a state-dependent noise. On the other hand in [4], the inflow process is constant but still with state-dependent times and magnitudes of the crashes. In all these papers, the main statistical characteristics of the systems at stationarity are computed.

To a large extent, the Markov chain model under study here is the simplest possible in the vast family of growth-collapse models. Using ideas stemming from excursion 
theory, we make precise here to what extent growth is (algebraically) slow when catastrophic events are rare under our over-simplistic model hypothesis. It is hoped that models in the same class of universality could share similar behaviors as one reasonably can expect some sort of robustness.

\section{The Model: A special Markov chain with Catastrophe on $\mathbb{Z}_{+}$}

Before proceeding with the MC model with holding probabilities, let us first investigate the simpler case where holding probabilities are absent. Let $\gamma>0$. We shall first consider the following discrete-time homogeneous Markov chain $X:=$ $\left(X_{n} ; n \geq 0\right)$ with state-space $\mathbb{Z}_{+}=\{0,1, \ldots\}$ and transition probabilities characterized by:

- given $X_{n}=x \in\{1,2, \ldots\}$, the increment of $X_{n}$ is

$$
\begin{aligned}
& +1 \text { with probability }: p_{x}=e^{-\gamma / x} \\
& -x \text { with probability }: q_{x}=1-e^{-\gamma / x} \sim \gamma / x .
\end{aligned}
$$

- given $X_{n}=0$, the increment of $X_{n}$ is +1 with probability $p_{0}=1$.

In other words, with $\left(U_{n}, n \geq 1\right)$ a sequence of independent identically distributed (i.i.d.) uniform random variables (rvs), the dynamics of $X_{n}$ reads

$$
X_{n+1}=\left(X_{n}+1\right) 1\left(U_{n+1}>q_{X_{n}}\right) .
$$

This irreducible Markov chain (MC) is in the class of general MCs with catastrophes (whose transition probabilities are state-dependent). It is reflected at the origin. In this model, the walker $X_{n}$ is occasionally bounced back to the origin. When $x$ is large, the drift of this MC is of order $1-\gamma-\gamma / x$. So when $\gamma>1$, the walker is attracted to the origin: The strength of the attraction goes like $\gamma-1$ for large $x$. For $\gamma<1$, the walker is repelled from the origin correspondingly. When $\gamma=1$, its drift is still attracting but of order $-1 / x$, and the drift that the walker feels vanishes when $x$ approaches $\infty$. We will see that while crossing the critical value $\gamma=1$ from above, the process $X$ switches from positive recurrent to null-recurrent.

In the context of machine replacement in reliability theory, one may classically interpret this MC as follows: at time $n=0$, a machine is put into service. This machine has a random lifetime, say $\tau_{0,0}$. After $\tau_{0,0}$, a new machine (with lifetime a copy of $\left.\tau_{0,0}\right)$ is installed to replace the old defective one and so on. The failure epochs of the successive machines constitute a renewal process on $\mathbb{N}_{0}$ generated by $\tau_{0,0}-1$. In this context, $X_{n}$ clearly represents the age of the machine currently in action (the time till the last machine failed before it was replaced by the new one in action at time $n$ ). Given the age of the current machine is $x$, there is an age-dependent probability $p_{x}$ that the machine will survive one more time unit and a probability $q_{x}$ that it will not. Clearly, the law of $\tau_{0,0}$ is $\mathbb{P}\left(\tau_{0,0}=k+1\right)=\prod_{y=0}^{k-1} p_{y} q_{k}, k \geq 1$, in terms of the $p \mathrm{~s}$ and the $\tau_{0,0} \mathrm{~s}$ are seen to be the times between consecutive visits to 0 for $X$. While assuming, as we do, that $p_{0}=1$, we suppose that the new machine is put into service as soon as the old one fails (there is no latency waiting time). 
One may also interpret this MC as follows: $X_{n}$ represents the size of some growing population at time $n$. Given $X_{n}=x$, at the next time unit, a new element presents itself for possible integration to the clan. The population can then grow by one unit with size-dependent probability $p_{x}$, integrating normally the new element, so: $X_{n} \rightarrow X_{n+1}=X_{n}+1$ with probability $p_{x}$. But there is a 'chance' $q_{x}$ that the new element is a black sheep (a "terminator") at the contact of which the whole population will get decimated, so: $X_{n} \rightarrow X_{n+1}=0$ with probability $q_{x}$; the black sheep is then the only survivor in the next generation. Clearly the occurrence of a black sheep is a catastrophic renewal event.

The contamination (or collapse) probability $q_{x}$ may be a decreasing or an increasing function of the current size $x$. In the former case, large populations are getting more and more immune to black sheep and one expects that large population sizes will get stable. This is the case under study here with $q_{x}=1-e^{-\gamma / x} \underset{x \rightarrow \infty}{\sim} \gamma x^{-1}$. We will see that such a MC $X$ is always recurrent. In case for instance $q_{x} \underset{x \rightarrow \infty}{\sim} \gamma x^{-\alpha}$ $(\alpha, \gamma>0)$, we would check that $X$ is transient if $\alpha>1$, positive recurrent if $\alpha<1$, whereas if $\alpha=1, X$ is positive recurrent if $\gamma>1$, null recurrent if $\gamma \leq 1$.

In the latter case, large populations are more susceptible and vulnerable to black sheep and so quite unlikely to grow large and develop too much. Think of a forest fire occasionally destroying completely an otherwise regularly growing forest. This would be the case for a model with $q_{x}=1-e^{-\gamma x}$. If one also thinks of the process of building a house of cards, clearly adding a new card to a house of cards of size $x$ is more likely to lead to a collapse of the whole structure if $x$ is already large.

Including holding probabilities. An important ingredient of our model is the opportunity to include holding probabilities, making the chain's changes algebraically rare. Let $\lambda \geq 0$. We shall in fact study the following more general Markov chain $\tilde{X}$, which may be viewed as a time-changed of $X$ if $\lambda>0$ :

- given $\widetilde{X}_{n}=x \in\{1,2, \ldots\}$, the increment of $\widetilde{X}_{n}$ is

$$
\begin{gathered}
+1 \text { with probability : } \widetilde{p}_{x}=x^{-\lambda} e^{-\gamma / x} \\
0 \text { with probability : } \widetilde{r}_{x}=1-x^{-\lambda} \\
-x \text { with probability : } \widetilde{q}_{x}=x^{-\lambda}\left(1-e^{-\gamma / x}\right) \sim \gamma / x^{\lambda+1} .
\end{gathered}
$$

- given $\widetilde{X}_{n}=0$, the increment of $\widetilde{X}_{n}$ still is +1 with probability $p_{0}=1$.

Clearly now, the dynamics of $\widetilde{X}_{n}$ reads

$$
\widetilde{X}_{n+1}=\widetilde{X}_{n} 1\left(U_{n+1} \leq \widetilde{r}_{X_{n}}\right)+\left(\widetilde{X}_{n}+1\right) 1\left(\widetilde{r}_{\widetilde{X}_{n}}<U_{n+1} \leq \widetilde{p}_{\widetilde{X}_{n}}+\widetilde{r}_{\widetilde{X}_{n}}\right) .
$$

When $x$ is large, the holding probability $\widetilde{r}_{x}$ is close to 1 if $\lambda>0$ so the chain $\tilde{X}$ remains steady with large probability. Whenever $\widetilde{X}$ moves, with large probability $e^{-\gamma / x}$, it moves up by one unit and only exceptionally, with probability $1-e^{-\gamma / x} \sim$ $\gamma / x$, does the catastrophic event take place. In this context, $\widetilde{X}_{n}$ is just the size of the cluster since the last catastrophe. This model therefore accounts for the lazy growth of stable large populations when the events leading either to growth or to collapse are themselves getting rare. 


\section{First properties of the General CAtAStrophe Markov Chain}

In this Section, we first supply a detailed study of the general catastrophe Markov chain, without specifying the catastrophe probabilities $q_{x}$.

4.1. Without holding probabilities. Consider a general catastrophe process $X_{n}$ reflected at the origin $\left(p_{0}=1\right.$ and $\left.q_{0}=0\right)$ for which both $p_{x}$ and $q_{x}>0$, for all $x \geq 1$, with $p_{x}+q_{x}=1$ and so with associated stochastic transition matrix: $P=[P(x, y)],(x, y) \in \mathbb{Z}_{+}^{2}$ with $P(x, 0)=q_{x}$ and $P(x, x+1)=p_{x}, x \geq 1$.

Existence and shape of the invariant measure. Let $\boldsymbol{\pi}^{\prime} \equiv\left(\pi_{0}, \pi_{1}, ..\right)$ be the row-vector of the invariant measure, whenever it exists. Then $\boldsymbol{\pi}$ should solve $\boldsymbol{\pi}^{\prime}=\boldsymbol{\pi}^{\prime} P$, whose formal solution is:

$$
\pi_{0}=\sum_{x \geq 1} \pi_{x} q_{x} \text { and } \pi_{x}=\pi_{0} \prod_{y=0}^{x-1} p_{y}, x \geq 1 .
$$

Let $u_{x}=\prod_{y=0}^{x-1} p_{y}$. Using the second equation, the first equation is satisfied whenever

$$
\sum_{x \geq 1} q_{x} \prod_{y=0}^{x-1} p_{y}=\sum_{x \geq 1}\left(u_{x}-u_{x+1}\right)=1,
$$

so also when $u_{\infty}=\prod_{y=1}^{\infty} p_{y}=0$ which is fulfilled iff $S_{2} \equiv \sum_{y=1}^{\infty} q_{y}=\infty$.

We first conclude that there exists an invariant measure iff $S_{2}=\infty$.

If in addition, $S_{1} \equiv \sum_{x \geq 1} \prod_{y=0}^{x-1} p_{y}<\infty$, then $\pi_{0}=\frac{1}{1+S_{1}} \in(0,1)$ and the invariant measure is unique and is a proper invariant probability measure. In this case, with the empty product being 1 , we have

$$
\pi_{x}=\frac{\prod_{y=0}^{x-1} p_{y}}{1+S_{1}}, x \geq 0
$$

When $S_{1}=\infty$, the measure solution to (2) exists but it is not a probability measure as its total mass $\pi_{0}\left(1+S_{1}\right)$ sums to infinity.

Return time to the origin. Let $X_{n}$ be the Markov chain with transition probability matrix $P$ and state-space $\mathbb{N}_{0}$. Starting from $X_{0}=x \geq 1$, the walker moves one step up with probability $p_{x}$ or goes back to the origin (the catastrophic event) with probability $q_{x}$ and then the process starts afresh from 0 . Clearly of interest are the times $\tau_{0,0}$ between consecutive visits to 0 (the first return times to 0 ) because if $X_{0}=0, X_{n}$ represents the backward recurrence time (the time separating $n$ to the previous visit to 0 ) of a discrete renewal process generated by $\tau_{0,0} \geq 2$. We have

$$
\mathbb{P}\left(\tau_{0,0}=k+1\right)=\prod_{y=0}^{k-1} p_{y} q_{k}=u_{k}-u_{k+1}, k \geq 1,
$$

which is also $\mathbb{P}\left(\tau_{0,0}>k\right)=\prod_{y=0}^{k-1} p_{y}=u_{k}$. 
Note that $p_{x}=\mathbb{P}\left(\tau_{0,0}>x+1\right) / \mathbb{P}\left(\tau_{0,0}>x\right)$ and $q_{x}=\mathbb{P}\left(\tau_{0,0}=x+1\right) / \mathbb{P}\left(\tau_{0,0}>x\right)$ : If the law of the lifetime $\tau_{0,0}$ is known in the first place, this gives the survival probability $p_{x}$ given the age (backward recurrence time) $X_{n}$ of the current machine is $x$.

Note also that $\mathbb{P}\left(\tau_{0,0}<\infty\right)$ iff $u_{\infty}=0\left(S_{2}=\infty\right)$ which is the recurrence condition for $X_{n}$.

We conclude that: If $S_{2} \equiv \sum_{y=1}^{\infty} q_{y}<\infty, X_{n}$ is transient. If $S_{2}=\infty, X_{n}$ is recurrent. If $S_{2}=\infty$ and $S_{1} \equiv \sum_{x \geq 1} \prod_{y=0}^{x-1} p_{y}<\infty, X_{n}$ is positive recurrent with $\mu:=\mathbb{E}\left(\tau_{0,0}\right)=1 / \pi_{0}=1+S_{1}<\infty$. If $S_{2}=S_{1}=\infty, X_{n}$ is null recurrent with $\tau_{0,0}<\infty$ almost surely (a.s.) and $\mathbb{E}\left(\tau_{0,0}\right)=\infty$. In the positive recurrent case,

$$
\pi_{x}=\frac{\prod_{y=0}^{x-1} p_{y}}{1+S_{1}}=\frac{\mathbb{P}\left(\tau_{0,0}>x\right)}{\mu}, x \geq 0
$$

which is the law of the limiting $(n \rightarrow \infty)$ backward recurrence time $X_{\infty}$ of the discrete renewal process generated by $\tau_{0,0}-1 \geq 1$. Let indeed $\tau_{n}$ be the length of the interval to which $n$ belongs. Then $\tau_{n} \stackrel{d}{\rightarrow} \tau$ (convergence in distribution) as $n \rightarrow \infty$ with

$$
\mathbb{P}(\tau=k)=\frac{k \mathbb{P}\left(\tau_{0,0}-1=k\right)}{\mu}, k \geq 1
$$

which is the size-biased version of the law of $\tau_{0,0}-1$. If $Y_{\infty}$ is the limiting $(n \rightarrow \infty)$ forward recurrence time of the discrete renewal process generated by $\tau_{0,0}-1 \geq 1$, with $X_{\infty}+Y_{\infty}=\tau$, we have $X_{\infty} \stackrel{d}{=} Y_{\infty}$ (distributional equality) and (see e.g. [20], Lemma 9, p. 447)

$$
\left(X_{\infty}, Y_{\infty}\right) \stackrel{d}{=}(U \circ \tau,(1-U) \circ \tau)
$$

Here, with $U$ a uniform random variable independent of $\tau, U \circ \tau$ is the $U$-thinning of $\tau: U \circ \tau=\sum_{l=1}^{\tau} B_{l}(U)$ where, given $U, B_{l}(U) ; l \geq 1$ are mutually independent and independent of $\tau$ rvs with law $\operatorname{Bernoulli}(U)$. Indeed

$$
\mathbb{P}(U \circ \tau=x)=\int_{0}^{1} \mathbb{P}(u \circ \tau=x) d u
$$

and

$$
\mathbb{P}(u \circ \tau=x)=\sum_{k \geq x}\left(\begin{array}{l}
k \\
x
\end{array}\right) u^{k}(1-u)^{k-x} \mathbb{P}(\tau=k) .
$$

Thus,

$$
\begin{aligned}
\mathbb{P}(U \circ \tau=x) & =\sum_{k \geq x}\left(\begin{array}{l}
k \\
x
\end{array}\right) \int_{0}^{1} u^{x}(1-u)^{k-x} d u \mathbb{P}(\tau=k) \\
& =\sum_{k \geq x} \frac{1}{k} \mathbb{P}(\tau=k)=\frac{1}{\mu} \mathbb{P}\left(\tau_{0,0}>x\right)=\pi_{x} .
\end{aligned}
$$

So there is a clear connection of our model with the discrete theory of renewal processes.

Time-reversal. It is of importance to check whether or not detailed balance holds for the MC under study here. Assume $X_{n}$ is recurrent. The catastrophe MC is 
not time-reversible as detailed balance does not hold. Let $Q \neq P$ be the transition matrix of the process $Y_{n}$ which is $X_{n}$ backward in time. With' denoting matrix transposition and $D_{\boldsymbol{\pi}}=\operatorname{diag}\left(\pi_{0}, \pi_{1}, \ldots\right)$, we have

$$
Q^{\prime}=D_{\pi} P D_{\pi}^{-1}
$$

It can be checked that the only non-null entries of $Q$ are its first row with $Q_{0,0}=$ $Q_{0,1}=0$ and $Q_{0, k}=\mathbb{P}\left(\tau_{0,0}=k\right)$ if $k \geq 2$ and the lower diagonal whose entries are ones. Starting from $Y_{0}=y$, the process $Y_{n}$ decays linearly till it hits 0 and once at state $0, Y_{n}$ jumps abruptly upward, undergoing a jump of amplitude $k \geq 2$ with probability $\mathbb{P}\left(\tau_{0,0}=k\right)$ before diminishing again and again to 0. Clearly, in case of positive recurrence, $Y_{n}$ models the forward recurrence time of the original process with $Y_{n} \stackrel{d}{\rightarrow} Y_{\infty}$ as $n \rightarrow \infty$. Note that $\boldsymbol{\pi}^{\prime}=\boldsymbol{\pi}^{\prime} Q(\boldsymbol{\pi}$ is also the invariant measure for $Q)$ and so: $X_{\infty} \stackrel{d}{=} Y_{\infty} \sim \pi_{x}$, as required.

\section{The scale (or harmonic) function.}

In the recurrent case, the sample paths of $X_{n}$ are made of i.i.d. excursions (the pieces of the sample paths between consecutive visits to 0). The lengths of the excursions are $\tau_{0,0}$. Let us look at their heights $H$. Clearly $H \stackrel{d}{=} \tau_{0,0}-1$, because $X$ grows linearly between consecutive visits to 0 . Let us rapidly check this with the use of the scale function. The scale function idea will appear more useful when we shall deal next with the time-changed version of the catastrophe MC because here the relationship between height and length of an excursion is far from trivial.

Assume $X_{0}=x$. Let $X_{n \wedge \tau_{x, 0}}$ stopping $X_{n}$ when it first hits 0 . Let us define the scale (or harmonic) function $\varphi$ of $X_{n}$ as the function which makes $Y_{n} \equiv \varphi\left(X_{n \wedge \tau_{x, 0}}\right)$ a martingale. The function $\varphi$ is important because, as is well-known, for all $0<x<$ $x_{*}$, with $\tau_{x}$ the first hitting time of $\left\{0, x_{*}\right\}$ starting from $x$

$$
\mathbb{P}\left(X_{\tau_{x}}=x_{*}\right)=\mathbb{P}\left(\tau_{x, x_{*}}<\tau_{x, 0}\right)=\frac{\varphi(x)}{\varphi\left(x_{*}\right)} .
$$

Using this remark, the event $H=h$ is realized when $\tau_{1, h}<\tau_{1,0}$ and $\tau_{h, h+1}>\tau_{h, 0}$, the latter two events being independent. Thus:

$$
\mathbb{P}(H=h)=\frac{\varphi(1)}{\varphi(h)}\left(1-\frac{\varphi(h)}{\varphi(h+1)}\right), h \geq 1 .
$$

We clearly have $\sum_{h \geq 1} \mathbb{P}(H=h)=1$ because partial sums are part of a telescoping series. Clearly, this is also $\mathbb{P}(H \geq h)=1 / \varphi(h)$. It remains to compute $\varphi$ with $\varphi(0)=0$. We wish to have: $\mathbb{E}_{x}\left(Y_{n+1} \mid Y_{n}=y\right)=y$, leading to

$$
\varphi(x)=p_{x} \varphi(x+1)+q_{x} \varphi(0)=p_{x} \varphi(x+1), x \geq 1 .
$$

Thus, the searched 'harmonic' function is

$$
\varphi(x)=\frac{1}{\prod_{y=0}^{x-1} p_{y}}, x \geq 1, \varphi(0) \equiv 0 .
$$

Note $\varphi(1)=1$. Equations (3) and (4) characterize the law of the excursion height of the random walker. Note

$$
\mathbb{P}(H \geq h)=1 / \varphi(h)=\prod_{y=0}^{h-1} p_{y}=\mathbb{P}\left(\tau_{0,0}>h\right) .
$$


showing, as expected from the beginning, that $H \stackrel{d}{=} \tau_{0,0}-1$.

Probability of extinction. Consider now the same Markov chain but assume now that $p_{0}=0, q_{0}=1$. In this case, the state 0 is absorbing. Consider then the restriction $\bar{P}$ of matrix $P$ to the states $\{1,2, \ldots\}$. Let $\phi_{x}, x \geq 1$ be the probabilities that state 0 is hit in finite time given the chain started originally at $x$. Let $\phi \equiv\left(\phi_{1}, \phi_{2}, . .\right)^{\prime}$ be the column-vector of these absorption probabilities. Let $\mathbf{q} \equiv\left(q_{1}, q_{2}, . .\right)^{\prime}$. Then $\boldsymbol{\phi}$ is the smallest non-negative solution to $\boldsymbol{\phi}=\mathbf{q}+\bar{P} \boldsymbol{\phi}$ whose formal solution is: $\phi=(I-\bar{P})^{-1} \mathbf{q}$. All $\phi_{x}$ can therefore be expressed in terms of $\phi_{1}$, leading simply to:

$$
1-\phi_{x}=\frac{1}{\prod_{y=1}^{x-1} p_{y}}\left(1-\phi_{1}\right) .
$$

If $S_{2} \equiv \sum_{y \geq 1} q_{y}=\infty$, then $u_{x}=\prod_{y=1}^{x-1} p_{y} \rightarrow 0$ : the restriction $\phi_{x} \in[0,1]$ forces $\phi_{1}=1$ and so $\phi_{x}=1$ for all $x<\infty$ : The state 0 is hit with probability 1 , starting from $x$, for all $x<\infty$. The Markov chain is recurrent.

But, if $S_{2}<\infty$, then we can take $\phi_{1}<1$ so long as $\phi_{x} \geq 0$ for all $x \geq 1$. The minimal solution occurs when $1-\phi_{1}=\mathbb{P}\left(\tau_{0,0}=\infty\right)=\prod_{y \geq 1} p_{y}>0$, leading to:

$$
\phi_{x}=1-\frac{\prod_{y \geq 1} p_{y}}{\prod_{y=1}^{x-1} p_{y}}=1-\prod_{y \geq x} p_{y} .
$$

In this case, $\phi_{x}<1$ for $x \geq 1$ and the absorbed random walker started at $x$ avoids 0 with positive probability (a transience case for the original reflected Markov chain). Note that $x<x^{\prime} \Rightarrow \phi_{x}>\phi_{x^{\prime}}$.

To summarize, we have:

Proposition 1. (i) If $S_{2}<\infty$, the $M C$ is transient and, with

$$
\tau_{x, 0}=\inf \left(n \geq 1: X_{n}=0 \mid X_{0}=x\right),
$$

$\mathbb{P}\left(\tau_{x, 0}=\infty\right)=\prod_{y \geq x} p_{y}>0$.

(ii) If $S_{2}=\infty$, the $M C$ is recurrent with $\mathbb{P}\left(\tau_{x, 0}=\infty\right)=0$. Moreover, it is: null recurrent if $S_{1}=\infty$, positive recurrent if $S_{1}<\infty$.

Due to irreducibility (because $p_{x}$ and $q_{x}>0$, for all $x \geq 1$ ), states are either all transient or recurrent.

Times to collapse. How long does it take, starting from $x \geq 1$, to first hit 0 ? We give here some insight on the way to compute the law of these first times to collapse. With $x \geq 1$, let thus $\tau_{x, 0}$ be the time it takes to first hit 0 , starting from $X_{0}=x \geq 1$. With $\tau_{x+1,0}^{\prime}$ a statistical copy of $\tau_{x+1,0}$, from first-step analysis, we clearly have:

$$
\tau_{x, 0} \stackrel{d}{=}\left(1-B_{x}\right) \cdot 1+B_{x} \cdot\left(1+\tau_{x+1,0}^{\prime}\right),
$$

where $B_{x}$ is a Bernoulli random variable with $\mathbb{P}\left(B_{x}=1\right)=p_{x}$. Therefore with $\phi_{x}(z)=\mathbb{E}\left(z^{\tau_{x, 0}}\right), \phi_{x}(z)$ obeys the recurrence $\phi_{x}(z)=q_{x} z+p_{x} z \phi_{x+1}(z)$, with initial condition $\phi_{1}(z)$ : again, $\phi_{x}(z)$ can easily be deduced once $\phi_{1}(z)$ is known. With $\phi_{0}(z)=\mathbb{E}\left(z^{\tau_{0,0}}\right)$ the probability generating function of the first return time to 
$0, \tau_{1,0} \stackrel{d}{=} \tau_{0,0}-1$ entails $\phi_{1}(z)=\phi_{0}(z) / z$. Because $\mathbb{P}\left(\tau_{0,0}=k+1\right)=\prod_{y=0}^{k-1} p_{y} q_{k}=$ $u_{k}-u_{k+1}$, with $u(z)=\sum_{k \geq 1} z^{k} u_{k}$, we have

$$
\phi_{1}(z)=\sum_{k \geq 1} z^{k} \prod_{y=0}^{k-1} p_{y} q_{k}=\sum_{k \geq 1} z^{k}\left(u_{k}-u_{k+1}\right)=\frac{z-1}{z} u(z)+1,
$$

fixing the initial condition. The full probability generating function (p.g.f.) of $\tau_{x, 0}$ follows.

With $\phi(z)=\left(\phi_{1}(z), \phi_{2}(z), \ldots\right)^{\prime}$ the column-vector of the $\phi_{x}(z)$, and $\mathbf{q}=\left(q_{1}, q_{2}, \ldots\right)^{\prime}$ the column-vector of the $q_{x}, \phi(z)$ solves:

$$
\phi(z)=z \mathbf{q}+z \bar{P} \boldsymbol{\phi}(z),
$$

whose formal solution is $\phi(z)=z(I-z \bar{P})^{-1} \mathbf{q}$, involving the resolvent of $\bar{P}$. When $z=1, \phi_{x}(1)=\phi_{x}=\mathbb{P}\left(\tau_{x, 0}<\infty\right)$ are the absorption probabilities already computed.

Transience versus recurrence. We here discuss the criterion for recurrence or transience of the general catastrophe Markov chain. When $S_{2}=\infty$, the recurrent chain started at $x$ first hits 0 with probability 1 and returns infinitely often to 0 . Given $X_{0}=x$, with $\mathcal{N}_{x, y} \equiv \sum_{n>0} \mathbf{1}\left(X_{n}=y\right)$, the number of visits to state $y$, then $\mathcal{N}_{x, y}=\infty, \mathbb{P}_{x}$-almost surely. If $\tau_{x, x}$ is the first return time at $x$, then $\mathbb{P}\left(\tau_{x, x}<\infty\right)=1$. Furthermore, with $\mathcal{N}_{x, y} \equiv \sum_{n=0}^{\tau_{x, x}} \mathbf{1}\left(X_{n}=y\right)$ the number of visits to state $y$ before the first return time to state $x$, then: $\mathbb{E}\left(\mathcal{N}_{x, y}\right)=\frac{\pi_{y}}{\pi_{x}}$ and by the Chacon-Ornstein limit ratio ergodic theorem:

$$
\frac{\sum_{n=0}^{N} \mathbf{1}\left(X_{n}=y\right)}{\sum_{n=0}^{N} \mathbf{1}\left(X_{n}=x\right)} \underset{N \nearrow \infty}{\rightarrow} \frac{\pi_{y}}{\pi_{x}}, \mathbb{P}_{x}-\text { almost surely. }
$$

Starting in particular from $x=0$, a recurrent chain is made of infinitely many independent and identically distributed (i.i.d.) excursions which are the sample paths of $\left(X_{n} ; n \geq 0\right)$ between consecutive visits to state 0 . We have: $\mathbb{E}\left(\mathcal{N}_{0, x}\right)=$ $\frac{\pi_{x}}{\pi_{0}}=\prod_{y=0}^{x-1} p_{y}$. When the chain is positive recurrent $\left(S_{1}<\infty\right)$ the expected time elapsed between consecutive visits to 0 is finite and equal to $\mathbb{E}\left(\tau_{0,0}\right) \equiv \mu=1 / \pi_{0}=$ $1+S_{1}$, whereas this expected time is infinite when the chain is null recurrent.

When $S_{2}=\infty$, the state $x \geq 0$ is transient. Thus, $\mathcal{N}_{x, x}<\infty, \mathbb{P}_{x}$-almost surely and $\mathbb{P}\left(\mathcal{N}_{x, x}=k\right)=\left(1-\alpha_{x}\right) \alpha_{x}^{k-1}$ where $\alpha_{x}=\mathbb{P}\left(\tau_{x, x}<\infty\right)<1$.

4.2. Including holding probabilities and time change. We now proceed with including the opportunity of a holding probability in the transition matrix of the catastrophe process.

Let $\rho_{x} \in(0,1), x \geq 1$. Consider now a general catastrophe MC $\widetilde{X}_{n}$ still reflected at the origin $\left(\widetilde{p}_{0}=1\right.$ and $\left.\widetilde{q}_{0}=0\right)$ and for which $\widetilde{p}_{x}=p_{x} \rho_{x}, \widetilde{q}_{x}=q_{x} \rho_{x}$ and $\widetilde{r}_{x}=1-\rho_{x}>0$, for all $x \geq 1$, with $\tilde{p}_{x}+\widetilde{q}_{x}+\widetilde{r}_{x}=1$. So $\widetilde{X}_{n}$ is generated by the stochastic transition matrix: $\widetilde{P}=[\widetilde{P}(x, y)],(x, y) \in \mathbb{Z}_{+}^{2}$ with $\widetilde{P}(x, 0)=\widetilde{q}_{x}$, $\widetilde{P}(x, x)=\widetilde{r}_{x}$ and $\widetilde{P}(x, x+1)=\widetilde{p}_{x}, x \geq 1$. There is now a positive holding probability $\widetilde{r}_{x}$ that given $\widetilde{X}_{n}=x \geq 1, \widetilde{X}_{n+1}=x$. The Markov chain $\widetilde{X}$ is lazy. 
In the context of machine replacement, the times at which $\widetilde{X}_{n}=\widetilde{X}_{n+1}=x$ are idle times, whereas in the population growth image, these times correspond to periods where no new candidate present themselves for possible integration to the current cluster (so in particular with no risk of appearance of the black sheep): the collapse is delayed. $\widetilde{X}_{n}$ accounts for the population size since the last catastrophe.

Invariant measure. We now investigate the way the invariant measure is modified by the adjunction of holding probabilities. Let $D_{\boldsymbol{\rho}}=\operatorname{diag}\left(\rho_{0}:=1, \rho_{1}, \rho_{2}, \ldots\right)$. We have $\widetilde{P}=I+D_{\boldsymbol{\rho}}(P-I)$. Let $\widetilde{\boldsymbol{\pi}}$ be the invariant measure associated to $\widetilde{P}$, when it exists. It should solve $\widetilde{\boldsymbol{\pi}}^{\prime}=\widetilde{\boldsymbol{\pi}}^{\prime} \widetilde{P}$, and we get:

$$
\widetilde{\pi}_{0}=\sum_{x \geq 1} \widetilde{\pi}_{x} q_{x} \rho_{x} \text { and } \widetilde{\pi}_{x}=\widetilde{\pi}_{0} \frac{\prod_{y=0}^{x-1} p_{y}}{\rho_{x}}, x \geq 1 .
$$

Using the second equation, the first equation is satisfied whenever

$$
\sum_{x \geq 1} q_{x} \rho_{x} \frac{\prod_{y=0}^{x-1} p_{y}}{\rho_{x}}=\sum_{x \geq 1}\left(u_{x}-u_{x+1}\right)=1,
$$

so again when $u_{x}=\prod_{y=1}^{x-1} p_{y} \underset{x \rightarrow \infty}{\rightarrow} 0$ which is fulfilled iff $S_{2} \equiv \sum_{y=1}^{\infty} q_{y}=\infty$ (the recurrence condition for $X$ ): The time change leading from $X$ to $\tilde{X}$ does not change the road map of $X$ so the recurrence criteria are identical for both $X$ and $\widetilde{X}$. However, the criteria for positive recurrence are modified.

Indeed, if in addition $\widetilde{S}_{1} \equiv \sum_{x \geq 1} \rho_{x}^{-1} \prod_{y=0}^{x-1} p_{y}<\infty$, then $\widetilde{\pi}_{0}=\frac{1}{1+\widetilde{S}_{1}} \in(0,1)$ and then the $\mathrm{MC}$ is positive recurrent with invariant probability measure

$$
\widetilde{\pi}_{x}=\widetilde{\pi}_{0} \frac{\prod_{y=0}^{x-1} p_{y}}{\rho_{x}}, x \geq 0
$$

Else if $\widetilde{S}_{1}=\infty$, the MC is null recurrent.

Scale function. $\widetilde{X}_{n}$ is again the size at time $n$ of the current population before the last catastrophic event. In the recurrent case $\left(S_{2}=\infty\right), \widetilde{X}$ is again made of i.i.d. excursions, whose height $\widetilde{H}$ has the same law as the one for $X$ : indeed, one can easily check that the scale function $\widetilde{\varphi}$ of $\widetilde{X}$ solving $\widetilde{P} \widetilde{\varphi}=\widetilde{\varphi}, \widetilde{\varphi}_{0}=0$, coincide with the scale function $\varphi$ of $X$ solving $P \varphi=\varphi, \varphi_{0}=0$. Because the scale function determines the height's law, we have the claimed statement that the height's law is left unchanged by time substitution.

Excursions lengths. However, because of the time change, the times $\widetilde{\tau}_{0,0}$ between consecutive visits to 0 (the excursions lengths of $\widetilde{X}$ ) are of course very different from $\tau_{0,0}$ (statistically longer). With $\widetilde{X}_{0}=0$, both times are related by

$$
\tau_{0,0}=\sum_{n=1}^{\tilde{\tau}_{0,0}} 1\left(\widetilde{X}_{n} \neq \widetilde{X}_{n-1}\right) .
$$


We thus have

$$
\mathbb{P}\left(\tau_{0,0}=k\right)=\sum_{l \geq k} \mathbb{P}\left(\widetilde{X}_{l-1}=k, \widetilde{\tau}_{0,0}=l\right),
$$

where, on the event $\widetilde{\tau}_{0,0}=l, \widetilde{X}_{l-1}$ is the height $\widetilde{H}_{l}$ of an excursion of length $l$ for $\widetilde{X}$.

Given the height of an excursion is $h$, we have

$$
\begin{aligned}
\mathbb{P}\left(\widetilde{\tau}_{0,0}=l \mid \widetilde{H}=h\right) & =q_{h} \mathbb{P}\left(\widetilde{\tau}_{0, h}=l-1 \mid \widetilde{\tau}_{0, h}<\widetilde{\tau}_{0,0}\right) \\
& =q_{h} \mathbb{P}_{0}\left(\widetilde{X}_{l-1}=h \mid \widetilde{\tau}_{1, h}<\widetilde{\tau}_{1,0}\right) .
\end{aligned}
$$

The p.g.f. of $\widetilde{\tau}_{0,0}$ can be obtained as follows. With $x \geq 1$, let $\widetilde{\tau}_{x, 0}$ be the time it takes for $\widetilde{X}$ to first hit 0 , starting from $\widetilde{X}_{0}=x \geq 1$. Therefore with $\widetilde{\phi}_{x}(z)=\mathbb{E}\left(z^{\widetilde{\tau}_{x, 0}}\right)$, $\widetilde{\phi}_{x}(z)$ obeys the recurrence $\widetilde{\phi}_{x}(z)=\widetilde{q}_{x} z+\widetilde{r}_{x} z \widetilde{\phi}_{x}(z)+\widetilde{p}_{x} z \widetilde{\phi}_{x+1}(z)$, with initial condition $\widetilde{\phi}_{1}(z)$ : again, $\widetilde{\phi}_{x}(z)$ can easily be deduced once $\widetilde{\phi}_{1}(z)$ is known. With $\widetilde{\phi}(z)=\left(\widetilde{\phi}_{1}(z), \widetilde{\phi}_{2}(z), \ldots\right)^{\prime}$ the column-vector of the $\widetilde{\phi}_{x}(z)$, and $\widetilde{\mathbf{q}}=\left(\widetilde{q}_{1}, \widetilde{q}_{2}, \ldots\right)^{\prime}$ the column-vector of the $\widetilde{q}_{x}, \widetilde{\phi}(z)$ solves:

$$
\widetilde{\phi}(z)=z \widetilde{\mathbf{q}}+z \widetilde{\widetilde{P}} \widetilde{\phi}(z),
$$

whose formal solution is $\widetilde{\phi}(z)=z(I-z \widetilde{\widetilde{P}})^{-1} \widetilde{\mathbf{q}}$, involving the resolvent of $\widetilde{\widetilde{P}}$. In particular, with $\mathbf{e}_{1}^{\prime}=(1,0,0, \ldots)$, the exact p.g.f. of $\widetilde{\tau}_{0,0}$ is:

$$
\widetilde{\phi}_{0}(z)=\mathbb{E}\left(z^{\widetilde{\tau}_{0,0}}\right)=z \widetilde{\phi}_{1}(z)=z^{2} \mathbf{e}_{1}^{\prime}(I-z \widetilde{\widetilde{P}})^{-1} \widetilde{\mathbf{q}}
$$

Note that $\overline{\widetilde{P}}$ is an upper-diagonal matrix with upper-diagonal entries $\widetilde{p}_{x}$ and diagonal entries $\widetilde{r}_{x}$. So this matrix is diagonable with eigenvalues $\widetilde{r}_{x}=1-\rho_{x}$. From this fact, in principle

$$
\widetilde{\phi}_{0}(z)=\sum_{x \geq 1} A_{x} \frac{z^{2}\left(1-\widetilde{r}_{x}\right)}{1-z \widetilde{r}_{x}}
$$

showing that $\widetilde{\tau}_{0,0}$ is an infinite mixture of (shifted) geometrically distributed rvs with success probabilities $\widetilde{r}_{x}$.

Time reversal. We now show how to compute the transition matrix of the timereversed process corresponding to the catastrophe MC with holding probabilities. When $\widetilde{X}_{n}$ is recurrent, this catastrophe MC is not time-reversible either. Let $\widetilde{Q} \neq \widetilde{P}$ be the transition matrix of the process $\widetilde{Y}_{n}$ which is $\widetilde{X}_{n}$ backward in time. $\widetilde{Y}$ now models a population that shrinks slowly and continuously to 0 before undergoing an instantaneous rebirth allowing the process to start afresh. Thus $\widetilde{Y}_{n}$ represents the size of the disintegrating cluster at time $n$ till the next forthcoming rebirth event. With ' denoting matrix transposition and $D_{\widetilde{\pi}}=\operatorname{diag}\left(\widetilde{\pi}_{0}, \widetilde{\pi}_{1}, \ldots\right)$, we indeed have

$$
\widetilde{Q}^{\prime}=D_{\widetilde{\pi}} \widetilde{P} D_{\widetilde{\pi}}^{-1}
$$

The only non-null entries of $\widetilde{Q}$ are its first row with $\widetilde{Q}_{0, k}=Q_{0, k}$ if $k \geq 1\left(\widetilde{Q}_{0,0}=0\right)$, the lower diagonal whose entries are $\rho_{k}, k \geq 1$ and the diagonal terms $\widetilde{Q}_{k, k}=1-\rho_{k}$, $k \geq 1$. Starting from $\widetilde{Y}_{0}=y$, the process $\widetilde{Y}_{n}$ decays linearly but with steady periods, 
till it hits 0 and once at state $0, \widetilde{Y}_{n}$ jumps abruptly upward, undergoing a jump of amplitude $k \geq 1$ with probability $\widetilde{Q}_{0, k}=Q_{0, k}$ before shrinking again to 0 .

\section{The special Catastrophe Markov Chain}

We now specify the results of the latter Section to the special MC defined in (1). The special MC deserves special interest in particular because it is, to a large extent, amenable to exact analytic computations. We shall limit ourselves to the study of $\widetilde{X}$ with the two parameters $\gamma>0, \lambda \geq 0$ because the study of $X$ (without holding probabilities) can be obtained from the one of the time-changed $\widetilde{X}$ while plugging $\lambda=0$.

Consider then the special MC $\widetilde{X}$, so determined by:

$$
\widetilde{p}_{x}=x^{-\lambda} e^{-\gamma / x} \text { and } \widetilde{q}_{x}=x^{-\lambda}\left(1-e^{-\gamma / x}\right), x \geq 1
$$

and satisfying $\widetilde{p}_{0}=1$ and $\widetilde{q}_{0}=0$ (reflection at the origin). Given $\widetilde{X}_{n}=x$, the local drift at $x$ of this MC is $f(x)=\widetilde{p}_{x}-x \widetilde{q}_{x}$. For all $0<\gamma \neq 1, \lambda>0$, this drift tends to 0 as $x$ tends to $\infty$ as a result of $f(x) \sim-(\gamma-1) x^{-\lambda}$. The asymptotic drift is $<0$ (respectively $>0$ ) when $\gamma>1$ (respectively $\gamma<1$ ). In the former (latter) case, $\widetilde{X}_{n}$ is asymptotically attracted (repelled) by the wall. When $\gamma=1, f(x) \sim-x^{-(\lambda+1)}$. Its local variance at $x$ is $g^{2}(x)=\widetilde{p}_{x}+x^{2} \widetilde{q}_{x}-\left(\widetilde{p}_{x}-x \widetilde{q}_{x}\right)^{2}$, with $g^{2}(x) \sim \gamma x^{1-\lambda}$. If $\lambda<1$ (respectively $\lambda>1$ ), the volatility at large $x$ diverges (vanishes) whereas $g^{2}(x) \sim \gamma$ if $\lambda=1$.

Both MCs $X$ and $\widetilde{X}$ are always recurrent because, with $q_{y} \sim \gamma / y, S_{2}=\sum_{y} q_{y}=\infty$. There is no transience case for the special MC. The invariant measure of $\tilde{X}$ satisfies

$$
\tilde{\pi}_{x} \sim \tilde{\pi}_{0} x^{-(\gamma-\lambda)},
$$

suggesting that $\tilde{\boldsymbol{\pi}} \equiv\left(\widetilde{\pi}_{x} ; x \in \mathbb{Z}_{+}\right)$is a proper (summable) probability measure if and only if $\gamma-\lambda>1$. Using the results of the latter Section, we thus get

Proposition 2. When $\gamma-\lambda \leq 1$, the $M C \widetilde{X}$ is null recurrent.

When $\gamma-\lambda>1$, the $M C \tilde{X}$ is positive recurrent; it has a unique and summable invariant measure satisfying $\widetilde{\pi}_{x} \underset{x \sim_{\infty}}{\sim} x^{-(\gamma-\lambda)}$. It is given explicitly by

$$
\widetilde{\pi}_{0}=\frac{1}{1+\widetilde{S}_{1}}=\frac{1}{1+\sum_{x \geq 1} x^{\lambda} \prod_{y=0}^{x-1} e^{-\gamma / y}}
$$

and

$$
\widetilde{\pi}_{x}=\widetilde{\pi}_{0} \frac{\prod_{y=0}^{x-1} p_{y}}{\rho_{x}}=\widetilde{\pi}_{0} x^{\lambda} \prod_{y=0}^{x-1} e^{-\gamma / y}, x \geq 1 .
$$

When $\gamma-\lambda>2$,

$$
\mathbb{E}\left(\widetilde{X}_{\infty}\right)=\sum_{x \geq 1} x \widetilde{\pi}_{x}<\infty
$$

so that the invariant measure has a finite explicit mean. 
Note that when $\gamma-\lambda>1$ (positive recurrence), the invariant probability measure is heavy-tailed with power-law exponent $\gamma-\lambda>1$. So $\widetilde{X}_{\infty}$ can get large which is a hint that large population sizes are stable as a result of $q_{x} \sim \gamma / x$, decreasing with $x$.

Excursion heights. The scale or 'harmonic' function of $\widetilde{X}$ is

$$
\widetilde{\varphi}(x)=\frac{1}{\prod_{y=0}^{x-1} p_{y}} \sim x^{\gamma}, x \geq 1, \widetilde{\varphi}(0) \equiv 0 .
$$

Therefore, the excursion heights of $\widetilde{X}$ have power-law tails

$$
\mathbb{P}(\widetilde{H} \geq h)=1 / \varphi(h) \sim h^{-\gamma} .
$$

Excursion lengths. In order to estimate the lengths of a big excursion, we suggest the following heuristics: Consider an excursion whose length $k$ and height $h$ are both assumed to be very large. We wish to estimate the height $h=h(k)$ for which the conditional probability $\mathbb{P}\left(\widetilde{\tau}_{0,0}=k \mid \widetilde{H}=h\right)$ is maximal, with:

$$
\begin{aligned}
\mathbb{P}\left(\widetilde{\tau}_{0,0}=k \mid \widetilde{H}=h\right) & =q_{h} \mathbb{P}\left(\widetilde{\tau}_{0, h}=k-1 \mid \widetilde{\tau}_{0, h}<\widetilde{\tau}_{0,0}\right) \\
& =q_{h} \mathbb{P}_{0}\left(\widetilde{X}_{k-1}=h \mid \widetilde{\tau}_{1, h}<\widetilde{\tau}_{1,0}\right) .
\end{aligned}
$$

We have

$$
\mathbb{P}\left(\widetilde{\tau}_{0,0}=k \mid \widetilde{H}=h\right) \approx q_{h} \prod_{l=1}^{k-1}\left(1-q_{x_{l}}\right)
$$

where $x_{l}$ is the position at time $l$ of the walker $\widetilde{X}$ which can roughly be estimated to be of order $x_{l} \approx l \frac{h}{k}$. For large $x, q_{x}$ is small and of order $\gamma x^{-(\lambda+1)}$. Thus

$$
\begin{gathered}
\mathbb{P}\left(\widetilde{\tau}_{0,0}=k \mid \widetilde{H}=h\right) \approx h^{-(\lambda+1)}\left(1-\gamma \sum_{l=1}^{k}\left(x_{l}\right)^{-(\lambda+1)}\right) \approx \\
h^{-(\lambda+1)}\left(1-\gamma\left(\frac{h}{k}\right)^{-(\lambda+1)} \sum_{l=1}^{k} l^{-(\lambda+1)}\right) \approx h^{-(\lambda+1)}\left(1-\gamma k h^{-(\lambda+1)}\right)
\end{gathered}
$$

Maximizing this over $h$ we get $h(k) \approx k^{1 /(\lambda+1)}$ suggesting that the height of a big excursion should scale like its length raised to the power $1 /(\lambda+1)$. Plugging $h(k)$ in the latter expression

$$
\mathbb{P}\left(\widetilde{\tau}_{0,0}=k \mid \widetilde{H} \geq h(k)\right) \approx k^{-1} .
$$

Thus

$$
\mathbb{P}\left(\widetilde{\tau}_{0,0}=k\right) \approx \mathbb{P}\left(\widetilde{\tau}_{0,0}=k, \widetilde{H} \geq h(k)\right) \approx k^{-1} h(k)^{-\gamma}=k^{-(\gamma /(\lambda+1)+1)} .
$$

Scaling properties. We now describe the scaling relationship between height and length of an excursion. Because $\mathbb{P}(\widetilde{H} \geq h) \sim h^{-\gamma}$, we conclude that the excursions 
lengths should also display power-law tails with

$$
\mathbb{P}\left(\widetilde{\tau}_{0,0}>k\right) \sim k^{-\gamma /(\lambda+1)} .
$$

Thus $\widetilde{\tau}_{0,0}$ and $\widetilde{H}^{\lambda+1}$ are expected to be tail equivalent with $\mathbb{P}\left(\widetilde{\tau}_{0,0} \geq k\right) \approx \mathbb{P}\left(\widetilde{H}^{\lambda+1} \geq k\right)$, meaning that the ratio $\mathbb{P}\left(\widetilde{\tau}_{0,0} \geq k\right) / \mathbb{P}\left(\widetilde{H}^{\lambda+1} \geq k\right)$ should tend to some constant when $k \rightarrow \infty$.

Recall that the exact p.g.f. of $\widetilde{\tau}_{0,0}$ is:

$$
\widetilde{\phi}_{0}(z)=\mathbb{E}\left(z^{\widetilde{\tau}_{0,0}}\right)=z \widetilde{\phi}_{1}(z)=z^{2} \mathbf{e}_{1}^{\prime}(I-z \overline{\widetilde{P}})^{-1} \widetilde{\mathbf{q}}
$$

where $\overline{\widetilde{P}}$ is an upper-diagonal substochastic matrix with upper-diagonal entries $\widetilde{p}_{x}$ and diagonal entries $\widetilde{r}_{x}$. So this matrix is diagonable with eigenvalues $\widetilde{r}_{x}=1-\rho_{x}$. The spectral gap here is $\rho_{x}=x^{-\lambda} \rightarrow 0$ as $x \rightarrow \infty$, algebraically fast, so the tails of $\widetilde{\tau}_{0,0}$ are not expected to be geometric, rather they are power-law. We have

$$
\sum_{k} z^{k} \mathbb{P}\left(\widetilde{\tau}_{0,0}>k\right)=: \widetilde{\Phi}_{0}(z)=\frac{1-\widetilde{\phi}_{0}(z)}{1-z}=\frac{1}{z(1-z) h(z)},
$$

where $h(z) \underset{z \rightarrow 1}{\sim}(1-z)^{-a}$ and $a=\gamma /(\lambda+1)$. Thus $\widetilde{\Phi}_{0}(z) \underset{z \rightarrow 1}{\sim}(1-z)^{-(1-a)}$. From the Karamata theorem (see [15]), therefore

$$
\left[z^{k}\right] \widetilde{\Phi}_{0}(z)=\mathbb{P}\left(\widetilde{\tau}_{0,0}>k\right) \underset{k \rightarrow \infty}{\sim} k^{-a} / \Gamma(1-a) .
$$

Note that in the positive recurrent case $(\gamma-\lambda>1), a>1$ so $\widetilde{\tau}_{0,0}$ has finite mean, whereas in the null recurrent case, $a<1$, and $\mathbb{E}\left(\widetilde{\tau}_{0,0}\right)=\infty$. We shall return to this crucial point later.

First passage times. Let $\widetilde{\tau}_{x, y}$ be the first passage time at $y \neq x$ when the process is started at $x$. We wish here to briefly derive an exact formal formula for the law of $\widetilde{\tau}_{x, y}$, making use of the Green function of a MC. Let

$$
\widetilde{\phi}_{x, y}(z) \equiv \sum_{k=1}^{\infty} z^{k} \mathbb{P}\left(\widetilde{\tau}_{x, y}=k\right)
$$

be the generating function of the law of $\widetilde{\tau}_{x, y}$. Then, with

$$
g_{z}(x, y) \equiv \sum_{n=0}^{\infty} z^{n} \mathbb{P}_{x}\left(\widetilde{X}_{n}=y\right)=\sum_{n=0}^{\infty} z^{n} \widetilde{P}^{n}(x, y)=(I-z \widetilde{P})^{-1}(x, y)
$$

the generating function of $\widetilde{P}^{n}(x, y)$ (the Green potential function of the chain), using $\widetilde{P}^{n}(x, y)=\sum_{m=0}^{n} \mathbb{P}\left(\widetilde{\tau}_{x, y}=m\right) \widetilde{P}^{n-m}(y, y)$, we easily get the expression:

$$
\widetilde{\phi}_{x, y}(z)=\frac{g_{z}(x, y)}{g_{z}(y, y)} .
$$

In particular,

$$
\widetilde{\phi}_{x, 0}(z)=\frac{g_{z}(x, 0)}{g_{z}(0,0)} \text { and } \widetilde{\phi}_{0, x}(z)=\frac{g_{z}(0, x)}{g_{z}(x, x)}
$$

are the generating functions of $\widetilde{\tau}_{x, 0}$ and $\widetilde{\tau}_{0, x}$. 
The p.g.f. $\widetilde{\phi}_{x, x}(z)$ first-return time $\widetilde{\tau}_{x, x}$ to state $x$ satisfies

$$
\widetilde{\phi}_{x, x}(z)=\frac{g_{z}(x, x)-1}{g_{z}(x, x)}=1-\frac{1}{g_{z}(x, x)}
$$

where $g_{z}(x, x)=\sum_{n=0}^{\infty} z^{n} \mathbb{P}_{x}\left(\widetilde{X}_{n}=x\right)=\sum_{n=0}^{\infty} z^{n} \widetilde{P}^{n}(x, x)$ is the Green function at $x$.

\section{First REtURn times to 0 And CONTACT PROBABILITy AT 0}

First return times to 0 and contact probability at 0 are classically related to one another by renewal arguments. Let us here compute the contact probability at 0 making use of the first return time probability to the origin. With $|z|<1$, let

$$
\widetilde{\phi}_{0}(z)=\sum_{k \geq 1} z^{k} \mathbb{P}\left(\widetilde{\tau}_{0,0}=k\right)
$$

be the generating function of the first return time to zero probability. Let also

$$
\widetilde{u}_{0}(z)=1+\sum_{n \geq 1} z^{n} \mathbb{P}_{0}\left(\widetilde{X}_{n}=0\right)
$$

be the Green potential function of the chain at state $x=0$. As can easily be checked by renewal arguments, $\widetilde{u}_{0}(z)=1+\widetilde{u}_{0}(z) \widetilde{\phi}_{0}(z)$, showing that

$$
\widetilde{u}_{0}(z)=\frac{1}{1-\widetilde{\phi}_{0}(z)} \text { and } \widetilde{\phi}_{0}(z)=1-\frac{1}{\widetilde{u}_{0}(z)} .
$$

Because the Markov chain $\widetilde{X}_{n}$ is always recurrent, $\widetilde{u}_{0}(1)=1+\sum_{n \geq 1} \mathbb{P}_{0}\left(\widetilde{X}_{n}=0\right)=$ $\infty$.

Recalling $\mathbb{P}\left(\widetilde{\tau}_{0,0}>k\right) \sim k^{-\gamma /(\lambda+1)}$, by the Karamata's theorem, we get $\widetilde{\phi}_{0}(z) \underset{z \downarrow 1}{\sim}$ $1-(1-z)^{\gamma /(\lambda+1)}$. Thus

$$
\widetilde{u}_{0}(z) \underset{z \downarrow 1}{\sim}(1-z)^{-\gamma /(\lambda+1)}
$$

We conclude from singularity analysis and Karamata's theorem applied to $\widetilde{u}_{0}(z)$ that

- If $0<\gamma-\lambda<1$ (null recurrence):

$$
\mathbb{P}_{0}\left(\widetilde{X}_{n}=0\right) \underset{n \rightarrow \infty}{\sim} n^{-(1-\gamma /(\lambda+1))}
$$

with $1-\gamma /(\lambda+1) \in(0,1)$. The contact probability at 0 decays algebraically to 0 , with decay exponent $1-\gamma /(\lambda+1)$.

- If $\gamma-\lambda>1$ (positive recurrence):

$$
\mathbb{P}_{0}\left(\widetilde{X}_{n}=0\right) \underset{n \rightarrow \infty}{\rightarrow} \tilde{\pi}_{0}<\infty
$$

the mass at 0 of the invariant probability measure. In the positive recurrent setup, the return time to the origin only occurs in finite time $\left(\widetilde{\tau}_{0,0}<\infty\right.$ with probability $1)$. Recall also that $\mathbb{E}\left(\widetilde{\tau}_{0,0}\right)<\infty$. In fact, a more detailed study of the singularities of $\widetilde{\phi}_{0}(z)$ shows that $\widetilde{\tau}_{0,0}$ has no moment of order larger or equal than $\frac{\gamma}{\lambda+1}$. 


\section{EXCURSion statistics of the SPeCial Markov Chain: extreme value ANALYSIS HEURISTIC}

In this Section, we study the way the mean and median of both the empirical average and sample maximum grow, depending on the values of $(\gamma, \lambda)$. Because growth is rare and regularly obstructed by catastrophic events, we expect growth to be slow. In fact, we will show that growth is algebraic only, with exponents that we can identify in terms of the model parameters $(\gamma, \lambda)$.

Recall the height of an excursion obeys

$$
\mathbb{P}(\widetilde{H} \geq h)=1 / \varphi(h) \sim h^{-\gamma} .
$$

Therefore, $\mathbb{E}(\widetilde{H})=\infty$ when $\gamma \in(0,1], \mathbb{E}(\widetilde{H})<\infty$ as soon as $\gamma>1$ and $\mathbb{E}\left(\widetilde{H}^{2}\right)<\infty$ as soon as $\gamma>2$.

For the excursions lengths, we have

$$
\mathbb{P}\left(\widetilde{\tau}_{0,0}>k\right) \sim k^{-\gamma /(\lambda+1)} .
$$

Therefore $\mathbb{E}\left(\widetilde{\tau}_{0,0}\right)=\infty$ when $\gamma-\lambda \leq 1, \mathbb{E}\left(\widetilde{\tau}_{0,0}\right)<\infty$ as soon as $\gamma-\lambda>1$ and $\mathbb{E}\left(\widetilde{\tau}_{0,0}^{2}\right)<\infty$ as soon as $\gamma-\lambda>2$.

We will summarize these results as follows:

Proposition 3. With the scale function given by (10), the law of the excursion height of the special walker is exactly given by:

$$
\mathbb{P}(\widetilde{H} \geq h)=1 / \varphi(h),
$$

satisfying $\mathbb{P}(\widetilde{H} \geq h) \approx h^{-\gamma}$.

(i) when $\gamma-\lambda \in(0,1]$ and $\gamma \in(0,1]$, the special $M C$ is null recurrent with both $\mathbb{E}\left(\widetilde{\tau}_{0,0}\right)=\infty$ and $\mathbb{E}(\widetilde{H})=\infty$.

(ii) when $\gamma-\lambda \in(0,1]$ and $\gamma>1$, the special $M C$ is null recurrent with $\mathbb{E}\left(\widetilde{\tau}_{0,0}\right)=\infty$ but with $\mathbb{E}(\widetilde{H})<\infty$.

(iii) In the positive recurrent case $(\gamma-\lambda>1)$ the special $M C$ sample paths are made of infinitely many i.i.d. excursions with both $\mathbb{E}\left(\widetilde{\tau}_{0,0}\right)$ and $\mathbb{E}(\widetilde{H})<\infty$.

7.1. Height and length of the largest excursion: the positive recurrent case. (Height). The preceding informations allow to derive the following qualitative result about the maximal height $\mathcal{H}_{N}$ reached by time $N$ in the positive recurrent case $(\gamma-\lambda>1)$ : by time $N$, with $\mu=\mathbb{E}\left(\widetilde{\tau}_{0,0}\right)<\infty$ there are indeed $N / \mu$ i.i.d. excursions on average. Thus,

$$
\mathcal{H}_{N}=\max _{n=1, \ldots,[N / \mu]} \widetilde{H}^{(n)}
$$

where $\widetilde{H}^{(n)} \stackrel{d}{=} \widetilde{H}$ are i.i.d. with law governed by (15). Due to (17), there exists a sequence $h_{N}$ such that $\frac{N}{\mu} \mathbb{P}\left(\widetilde{H}>h_{N}\right) \rightarrow_{N} \nearrow_{\infty} \alpha$ for some $\alpha>0$, say $\alpha=\log 2$. 
From (15), we obtain:

$$
h_{N} \approx N^{1 / \gamma} .
$$

Using this, we have

$$
\mathbb{P}\left(\mathcal{H}_{N} \leq h_{N}\right)=\left(1-\mathbb{P}\left(\widetilde{H}>h_{N}\right)\right)^{N / \mu} \rightarrow_{N}{ }_{\infty} e^{-\alpha}=1 / 2,
$$

and therefore (when $\gamma-\lambda>1$ ), the typical (median) maximal height that the MC reaches by time $N$, grows like $N^{1 / \gamma}$. With $\mathbb{M}$ denoting the median value, with $M_{N}:=\max \left(\widetilde{X}_{1}, . ., \widetilde{X}_{N}\right)$, we therefore have: $\mathbb{M}\left(M_{N}\right)=h_{N} \approx N^{1 / \gamma}$.

Clearly, the number of $\widetilde{H}_{n}, n=1, . .,[N / \mu]$ exceeding $h_{N}$ converges to a Poisson $(\alpha)$

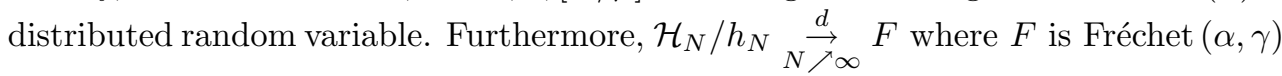
distributed with $\mathbb{P}(F \leq x)=e^{-\alpha x^{-\gamma}}$.

(Length). Similarly for the lengths, let

$$
\tau_{N}=\max _{n=1, . .,[N / \mu]} \widetilde{\tau}_{0,0}^{(n)}
$$

be the length of the largest excursion, with $\widetilde{\tau}_{0,0}^{(n)} \stackrel{d}{=} \widetilde{\tau}_{0,0}$ i.i.d. with law governed by (16). Due to (16), there exists a sequence of time lags $k_{N}$ such that $\frac{N}{\mu} \mathbb{P}\left(\widetilde{\tau}_{0,0}>k_{N}\right) \rightarrow_{N / \infty} \alpha>0$. Since $\mathbb{P}\left(\widetilde{\tau}_{0,0}>k\right) \approx k^{-\gamma /(\lambda+1)}$, we get:

$$
k_{N} \approx N^{(\lambda+1) / \gamma} \text {. }
$$

We have

$$
\mathbb{P}\left(\tau_{N} \leq k_{N}\right)=\left(1-\mathbb{P}\left(\widetilde{\tau}_{0,0}>k_{N}\right)\right)^{[N / \mu]} \rightarrow_{N} \gamma_{\infty} e^{-\alpha}=1 / 2 .
$$

Therefore, when $\gamma-\lambda>1$, the typical length of the excursion with maximal length by time $N$ grows like $N^{(\lambda+1) / \gamma} \ll N$. Note that

$$
h_{N} \approx k_{N}^{1 /(\lambda+1)},
$$

so that the typical height of the largest excursion scales like its length raised to the power $1 /(\lambda+1)$.

Note also that, observing $\mathbb{E}\left(M_{k_{N}}\right) \approx \mathbb{M}\left(M_{N}\right)$, we obtain

$$
\mathbb{E}\left(M_{N}\right) \approx \mathbb{M}\left(\max \left(\widetilde{X}_{1}, . ., \widetilde{X}_{k_{N}^{-1}}\right)\right)=\left(N^{\gamma /(\lambda+1)}\right)^{1 / \gamma}=N^{1 /(\lambda+1)}
$$

with $\mathbb{M}\left(M_{N}\right) \ll \mathbb{E}\left(M_{N}\right)$ in the positive-recurrent regime under study $\gamma-\lambda>1$.

Let $\tau_{0, h}$ be the first time at which some excursion height exceeds the level $h$ (the time between failure at $h$ ). We have

$$
\mathbb{P}\left(\tau_{0, h}>N\right)=\mathbb{P}\left(\mathcal{H}_{N} \leq h\right)=(1-\mathbb{P}(\widetilde{H}>h))^{[N / \mu]} .
$$

Due to (15), for all $\alpha>0$, assuming $h$ large, we get:

$$
\mathbb{P}\left(h^{-\gamma} \tau_{0, h}>\alpha\right) \underset{h \text { large }}{\sim}\left(1-h^{-\gamma}\right)^{\frac{\alpha}{\mu} h^{\gamma}} \rightarrow e^{-\frac{\alpha}{\mu}},
$$

showing that $\tau_{0, h}$ is of order $\mathbb{M}\left(\tau_{0, h}\right) \approx h^{\gamma}$ with an exponential limit law. This point is in accordance with (18). We can summarize these results as follows: 
Proposition 4. Assume $\gamma-\lambda>1$ (positive recurrence of the special walker). Then,

(i) the typical (median) height $h_{N}$ of its largest excursion satisfies $h_{N} \approx N^{1 / \gamma}$.

(ii) the typical (median) length $k_{N}$ of its largest excursion satisfies $k_{N} \approx N^{(\lambda+1) / \gamma}$, so with $h_{N} \approx k_{N}^{1 /(\lambda+1)}$.

(iii) the typical (median) time to failure at level $h$ satisfies: $\mathbb{M}\left(\tau_{0, h}\right) \approx h^{\gamma}$.

7.2. The null recurrent case. To some extent, this situation extends to the range $\gamma-\lambda<1$, although it deserves a special treatment. In this null recurrent case indeed, $\mu=\infty$ and so one deals with very large and therefore rare excursions (see [14]). By renewal arguments, the expected number of such excursions by time $N$ (large) now is of order $N^{a} / c$ (with $a=\gamma /(\lambda+1)<1$ and $c>0$ ), so much smaller than $N$. The typical length $k_{N}$ of the largest excursion by time $N$ is now given by:

$$
\frac{N^{a}}{c} \mathbb{P}\left(\widetilde{\tau}_{0,0}>k_{N}\right) \rightarrow_{N}>_{\infty} \alpha>0,
$$

leading to $k_{N} \approx N$ (with no dependence of the scaling exponent on $\gamma$ and $\lambda$ ): In this null-recurrent regime, the size of a typical excursion is the largest possible, corresponding to a single big excursion (or perhaps just a few of them).

Similarly, the maximal height $\mathcal{H}_{N}$ reached by time $N$ in this null recurrent case is again given by:

$$
\mathcal{H}_{N}=\max _{n=1, \ldots,\left[N^{a} / c\right]} \widetilde{H}^{(n)},
$$

where $H^{(n)}$ are i.i.d. with law governed by (15). Due to (10), there exists a sequence $h_{N}$ such that $\frac{N^{a}}{c} \mathbb{P}\left(\widetilde{H}>h_{N}\right) \rightarrow_{N}{ }_{\infty} \alpha$ for some $\alpha>0$, leading to

$$
h_{N} \approx N^{1 /(\lambda+1)} \text {. }
$$

Thus, when $\gamma-\lambda<1, h_{N} \approx k_{N}^{1 /(\lambda+1)}$. Although when $\gamma-\lambda<1, h_{N}$ and $k_{N}$ are not individually of the same order of magnitude as when $\gamma-\lambda>1$, the typical height of the largest excursion continues to scale like its length raised to the power $1 /(\lambda+1)$.

In the null recurrent case, we also have

$$
\mathbb{P}\left(\tau_{0, h}>N\right)=\mathbb{P}\left(\mathcal{H}_{N} \leq h\right)=(1-\mathbb{P}(\widetilde{H}>h))^{\left[N^{a} / c\right]} .
$$

Due to (15), for all $\alpha>0$, when $h$ is large, we get:

$$
\mathbb{P}\left(h^{-(\lambda+1)} \tau_{0, h}>\alpha\right) \underset{h \text { large }}{\sim}\left(1-h^{-\gamma}\right)^{\alpha^{a} h^{\gamma} / c} \rightarrow e^{-\alpha^{a} / c},
$$

showing that the time to failure $\tau_{0, h}$ at $h$ is now of order $\mathbb{M}\left(\tau_{0, h}\right) \approx h^{\lambda+1}$ with a Weibull limit law. We shall summarize these results as follows:

Proposition 5. Assume $\gamma-\lambda<1$ (null recurrence of the special walker). Then,

(i) the typical (median) height $h_{N}$ of its largest excursion satisfies $h_{N} \approx N^{1 /(\lambda+1)}$.

(ii) the typical (median) length $k_{N}$ of its largest excursion satisfies $k_{N} \approx N$, so still with $h_{N} \approx k_{N}^{1 /(\lambda+1)}$.

(iii) the typical (median) time to failure at level $h$ satisfies: $\mathbb{M}\left(\tau_{0, h}\right) \approx h^{\lambda+1}$. 
From this result concerning the null recurrent case and the previous one in the positive recurrent case, we observe that the typical height of the largest excursion always scales like its length raised to the power $1 /(\lambda+1)$.

7.3. Height and length of excursions. Scaling properties in the regime $1<\gamma-\lambda<2$. The previous study in the positive recurrent case, suggests that, at least when $1<\gamma-\lambda<2$, it could always be true that the expected height of any excursion (not only the largest) should scale like its length raised to the power $1 /(\lambda+1)$. If this were to be the case, defining the empirical mean of the walk as

$$
\bar{X}_{N} \equiv \frac{1}{N} \sum_{n=1}^{N} \tilde{X}_{n}
$$

then,

$$
\mathbb{E}\left(\bar{X}_{N}\right)=\frac{1}{N} \sum_{k=1}^{N} k \frac{N}{\mu} \mathbb{P}\left(\tau_{0,0}=k\right) k^{1 /(\lambda+1)},
$$

because there are approximately $\frac{N}{\mu} \mathbb{P}\left(\tau_{0,0}=k\right)$ excursions of length $k$ whose contribution to the empirical mean is $k \frac{N}{\mu} \mathbb{P}\left(\tau_{0,0}=k\right)$, each with typical height $k^{1 /(\lambda+1)}$. Using (11), we therefore expect that in the range $1<\gamma-\lambda<2$

$$
\begin{aligned}
\mathbb{E}\left(\bar{X}_{N}\right) & \approx \sum_{k=1}^{N} k^{1+1 /(\lambda+1)} \mathbb{P}\left(\tau_{0,0}=k\right) \approx \sum_{k=1}^{N} k^{1+1 /(\lambda+1)-\left(1+\frac{\gamma}{\lambda+1}\right)} \\
& =\sum_{k=1}^{N} k^{-\frac{\gamma-1}{\lambda+1}} \approx N^{1-\frac{\gamma-1}{\lambda+1}}=N^{\frac{\lambda-\gamma+2}{\lambda+1}} .
\end{aligned}
$$

This shows that the expected height of the special MC $\widetilde{X}$ at time $N$ grows like $N^{\frac{\lambda-\gamma+2}{\lambda+1}}$.

Note that we get a sub-diffusive (super-diffusive) regime in the domain $\{1<\gamma-\lambda<2\} \cap$ $\{\gamma<(\lambda+3) / 2\}$ (respectively $\{1<\gamma-\lambda<2\} \cap\{\gamma>(\lambda+3) / 2\})$. In particular if $\lambda>1$, the regime is always super-diffusive.

Rather considering the median value $\mathbb{M}\left(\bar{X}_{N}\right)$ of $\bar{X}_{N}$, still when $1<\gamma-\lambda<2$, we get

$$
\mathbb{M}\left(\bar{X}_{N}\right) \approx \sum_{k=1}^{k_{N}} k^{1+1 /(\lambda+1)} \mathbb{P}\left(\tau_{0,0}=k\right)=\sum_{k=1}^{k_{N}} k^{-\frac{\gamma-1}{\lambda+1}} \approx k_{N}^{1-\frac{\gamma-1}{\lambda+1}} \approx N^{\frac{\lambda-\gamma+2}{\gamma}},
$$

with $\mathbb{M}\left(\bar{X}_{N}\right) \ll \mathbb{E}\left(\bar{X}_{N}\right) \ll h_{N}=\mathbb{M}\left(M_{N}\right)$ for the parameter range $1<\gamma-\lambda<2$ under concern. The median height $\mathbb{M}\left(\bar{X}_{N}\right)$ of $\bar{X}_{N}$ can also be estimated in the following way:

$$
\mathbb{M}\left(\bar{X}_{N}\right) \approx \sum_{x=1}^{h_{N}} x \mathbb{P}\left(\bar{X}_{N}=x\right) \approx \sum_{x=1}^{h_{N}} x x^{-(\gamma-\lambda)} \approx h_{N}^{2+\lambda-\gamma} \approx N^{(2+\lambda-\gamma) / \gamma},
$$

where we used that when $N$ is large, $\mathbb{P}\left(\bar{X}_{N}=x\right)$ is 'close' to the invariant measure $\tilde{\boldsymbol{\pi}}$ which scales for large $x$ like $x^{-(\gamma-\lambda)}$. Indeed, with $\|P-Q\|_{T V} \equiv \sup _{A}\{P(A)-Q(A)\}$ defining the total variation distance between two probability measures $P$ and $Q$,

$$
\left\|\mathbb{P}\left(\bar{X}_{N}=\cdot\right)-\tilde{\pi} \cdot\right\|_{T V} \approx \mathbb{P}\left(X_{N} \geq h_{N}\right) \approx h_{N}^{1+\lambda-\gamma} \approx N^{-(\gamma-\lambda-1) / \gamma},
$$


from (18). The latter quantity $N^{-(\gamma-\lambda-1) / \gamma}$ tends to 0 because $\gamma-\lambda>1$. This heuristic approach also suggests that the convergence to equilibrium of $\mathbb{P}\left(\bar{X}_{N}=\right.$.) should be algebraically slow when $1<\gamma-\lambda<2$. To summarize, we obtained:

Proposition 6. Let $\bar{X}_{N}$ in (24) define the empirical mean height of the walker by time $N$.

(i) Assume $1<\gamma-\lambda<2$. Then, the expected value $\mathbb{E}\left(\bar{X}_{N}\right)$ of $\bar{X}_{N}$ satisfies: $\mathbb{E}\left(\bar{X}_{N}\right) \approx N^{(2+\lambda-\gamma) /(\lambda+1)}$. Its typical (median) value $\mathbb{M}\left(\bar{X}_{N}\right)$ satisfies: $\mathbb{M}\left(\bar{X}_{N}\right) \approx$ $N^{(2+\lambda-\gamma) / \gamma}$ with, since $\mathbb{M}\left(M_{N}\right) \approx N^{1 / \gamma}$ and $\mathbb{E}\left(M_{N}\right) \approx N^{1 /(\lambda+1)}$ :

$\mathbb{M}\left(\bar{X}_{N}\right) \ll \mathbb{E}\left(\bar{X}_{N}\right) \ll \mathbb{M}\left(M_{N}\right) \ll \mathbb{E}\left(M_{N}\right)$.

(ii) When $\gamma-\lambda>2, \mathbb{E}\left(\bar{X}_{N}\right) \rightarrow_{N / \infty} \mathbb{E}\left(\widetilde{X}_{\infty}\right)$, the finite mean of the invariant measure $\tilde{\boldsymbol{\pi}}$. However, $\mathbb{M}\left(M_{N}\right) \approx N^{1 / \gamma}$ and $\mathbb{E}\left(M_{N}\right) \approx N^{1 /(\lambda+1)}$ still holds true in this parameter range.

In $(i)$, it can be checked that the scaling exponent $(2+\lambda-\gamma) /(\lambda+1)$ of $\mathbb{E}\left(\bar{X}_{N}\right)$ is indeed smaller than the one $1 / \gamma$ of $\mathbb{M}\left(M_{N}\right)$ in the regime under study $1<$ $\gamma-\lambda<2$. Note also that one expects that, normalizing the maximum by its mean, $M_{N} / N^{1 /(\lambda+1)}$ should admit a limit law as $N \rightarrow \infty$.

(ii) is clear because, when $\gamma-\lambda>2, \bar{X}_{N} \underset{N \rightarrow \infty}{\rightarrow} \mathbb{E}\left(\widetilde{X}_{\infty}\right)<\infty$ almost surely.

\section{REFERENCES}

[1] Bak, P. How nature works. The science of self-organized criticality. Copernicus, New York, 1996.

[2] Bak, P., Christensen, K., Danon, L. and Scanlon, T. Unified scaling law for earthquakes, Phys. Rev. Lett., 88, (2002), p. 178501.

[3] Brockwell, P. J., Gani, J. M. and Resnick, S. I. Catastrophe processes with continuous statespace. Austral. J. Statist. 25 (1983), no. 2, 208-226.

[4] Boxma, O., Perry, D., Stadje, W. and Zacks, S. A Markovian growth-collapse model. Adv. in Appl. Probab. 38 (2006), no. 1, 221-243.

[5] Cairns, B., Pollett, P. K. Extinction times for a general birth, death and catastrophe process. J. Appl. Probab. 41 (2004), no. 4, 1211-1218.

[6] Carlson, J.M, Langer, J. S. and Shaw, B. E. Dynamics of earthquake faults. Rev. Mod. Phys. $66,(1994), 657-670$.

[7] Corral, A. Long-term clustering, scaling and universality in the temporal occurrence of earthquakes. Phys. Rev. Lett. 92 (10), (2004), p. 108501

[8] Eliazar, I., Klafter, J. A growth-collapse model: Lévy inflow, geometric crashes, and generalized Ornstein-Uhlenbeck dynamics. Phys. A 334 (2004), 1-21.

[9] Eliazar, I., Klafter, J. Stochastic Ornstein-Uhlenbeck capacitors. J. Stat. Phys. 118 (2005), $177-198$.

[10] Eliazar, I., Klafter, J. Growth-collapse and decay-surge evolutions, and geometric Langevin equations. Phys. A 367 (2006), 106-128.

[11] Fernandez, R., Ferrari, P. and Galves, A. Coupling, renewal and perfect simulation of chains of infinite order. http://www.univ-rouen.fr/LMRS/Persopage/Fernandez/notasfin.pdf.

[12] Ferrari, P., Galves, A. Construction of stochastic processes, coupling and regeneration. http://www.ime.usp.br/ pablo/book/oct2001/oct2001.pdf.

[13] Gripenberg, G. A stationary distribution for the growth of a population subject to random catastrophes. J. Math. Biol. 17 (1983), no. 3, 371-379.

[14] Huillet, T., Raynaud, H-F. On rare and extreme events. Chaos Solitons Fractals 12 (2001), no. $5,823-844$.

[15] Karamata, J.; Sur un mode de croissance régulière. Théorèmes fondamentaux. Bull. Soc. Math. France 61 (1933), 55-62. 
[16] Kella, O. On growth-collapse processes with stationary structure and their shot-noise counterparts. J. Appl. Probab. 46 (2009), no. 2, 363-371.

[17] Karlin, S., McGregor, J. Occupation time laws for birth and death processes. 1961 Proc. 4th Berkeley Sympos. Math. Statist. and Prob., Vol. II pp. 249-272 Univ. California Press, Berkeley, Calif.

[18] Martin, J. B. Batch queues, reversibility and first-passage percolation. Queueing Syst. 62 (2009), no. 4, 411-427.

[19] Robert, C. Y. Stochastic stability of some state-dependent growth-collapse processes. Adv. in Appl. Probab. 39 (2007), no. 1, 189-220.

[20] Steutel, F. W., van Harn, K. Infinite divisibility of probability distributions on the real line. Monographs and Textbooks in Pure and Applied Mathematics, 259. Marcel Dekker, Inc., New York, 2004.

[21] Stirzaker, D. Processes with catastrophes. Math. Sci. 31 (2006), no. 2, 107-118.

Laboratoire de Physique Théorique et Modélisation, CNRS-UMR 8089 et Université de Cergy-Pontoise, 2 Avenue Adolphe Chauvin, F-95302, Cergy-Pontoise, France, Thierry.Huillet@uCERGY.FR 